\title{
Research on Recommendation Operation Strategy of News and In- formation Products
}

\author{
Yuxi Liu* \\ Boshang Culture Media Co., Ltd. E-mail: liuyx@qq.com
}

\begin{abstract}
For China's news industry, personalized recommendation of news and information products is beginning to take off, but it is growing rapidly. Excess information content and limited time and energy of users urgently need common numbers to balance. It is not the ultimate goal to blindly provide users with instant news content as media. With the rising status of users, the times have higher requirements for media. In today's society, improving information processing ability is more important than the speed of information dissemination. Users are forbidden to keep copper in the information cage. Repeated bloody, violent, misleading and other information are distressing. How to solve information overload for news and information product operators? How to adjust operation strategy from the perspective of users? How to solve the relationship between technical algorithm and manual intervention? How can personalized recommendation promote the development of news industry?
\end{abstract}

Keywords: News Information; Individualization; Operational Strategy

Personalized recommendation is an inevitable trend under the development of technology. Algorithm recommendation and news industry have achieved mutual success, and major news information products have shouldered the banner of "personalized recommendation". From the users' point of view, the news content is distributed and disseminated. Under the background of Internet development, the number of Internet users has increased at a fission rate, and the news industry has begun to pay more attention to the audience's news and information needs. News and information products are the products of the integration of news industry and Internet in the new era. It is important to highlight the characteristics of the times, while maintaining the related attributes of news. For the operators of news and information products, how to make the products better adapt to the market is very important. Personalized news rec- ommendation is low in cost. Accurate delivery, which can enhance user stickiness and increase product exposure, is a service function that can not be ignored in the process of news dissemination. In the era of information explosion, the most interesting news information to users should be recommended at the most suitable time, which means that personalized news recommendation must make the connection between products and users, and constantly strengthen the connection.

\section{Overview of news information products and personalized news recommendation}

News information products are the display carrier of news personalized recommendation, which is the dis semination way of news information products, which

This is an open-access article distributed under the terms of the Creative Commons Attribution Non-Commercial License (http://creativecommons.org/licenses/by-nc/4.0/), which permits unrestricted non-commercial use, distribution, and reproduction in any medium, provided the original work is properly cited. 
complement each other and influence each other. Under the background of rapid development of mobile news, the immediacy of news itself and the demand of user information acquisition both determine the rationality of personalized recommendation of news information products to a certain extent. What kind of news information users want to get and what kind of news information products want to disseminate have been recommended more accurately under the blessing of algorithm technology. Individualized news recommendation has become the development trend of portal websites and aggregated news and information products, and will certainly continue to set off a wave.

\subsection{Overview of news and information products}

The concept of products is more abundant in the Internet age. The tangible or intangible carriers that can supply the market, be consumed and used by the audience and meet some needs of the audience can be called products. The new era has given new content to product concepts, and Internet products have become faster and more convenient with the development of network and communication. Extending from the traditional field to meet the needs of Internet users, this feature has also caused an uproar in the news media industry. News and information products are the inevitable trend of news media development in the new era and the backbone communication force that can not be ignored. Every Internet user is exposed to various kinds of information and receives news reports from different media sources every day. News and information products play the role of disseminator in today's information bombing. The competition among media is not only the competition between content and brand, but also the competition of user market. Different positioning and operation modes of news and information products determine the development of news and information products.

\subsection{Personalized news recommendation}

Personalized news recommendation is the most concentrated and competitive part in the operation of news information products. Traditional news media clients and news clients of portal websites want to develop, but at the same time, they also use their respective advantages to integrate personalized news recommenda- tion to meet users' information needs. Seizing the user market and preempting the data advantage are the two most important reasons for the emergence and prosperity of personalized news recommendation. Paying attention to the user market by major news media is a correct interpretation of the industry development situation in the Internet era, and will also provide good experience and enlightenment for the market rules and industry development of news and information products.

What is personalized recommendation? News recommendation has something in common with information recommendation in other fields, and its main types can be roughly divided into two categories, full recommendation and personalized recommendation; Full-volume news recommendation is the news recommendation behavior for all downloading users, that is, the content is recommended indiscriminately, and the receiving users are all downloading users. This type of recommendation method is mainly used in two situations. First, the news information products of traditional news media, without technical intervention, recommend for all users of news events; Second, the news recommendation based on a major hot event has a strong news value. But fundamentally, the final recommendation range is often less than the real download and installation number because of the user's behavior and restrictions. If the focus of full news lies in the news event content itself, the starting point of personalized recommendation lies in the different news demands of users. For example, the development of localized content recommendation as personalized news recommendation is the further research and consideration of users' behaviors and reading feelings by news information product operators. Different users come from different regions, and the mobile client installs GPS positioning for the location of users and recommends news information of specific regions, which can arouse readers' psychological identity and shorten the psychological distance with users. In the same way, any news that is related to interests or the news content that is most interested in the moment will enhance users' trust in the product itself, which has strong commercial value. For personalized recommendation, it is an indispensable part.

Therefore, among the two types of news recommendation, the emergence of personalized news recommendation is the awakening of news media and news 
information product operators' consciousness, and it is the emphasis on the fundamental user position of the Internet. Personalized recommendation relies on big data with the help of algorithms, and analyzes big data according to users' usage behaviors such as browsing, subscribing, collecting, praising, commenting and duration of social news information products. Meanwhile, users' portraits are analyzed across platforms in the same type of platform, so that users' behaviors can be known like the palm of their hand in silence, so as to make personalized news recommendation better. In addition to machine intervention, editors will also intervene in certain content. In view of the present development situation, the disadvantages of technology are becoming more and more obvious.

\section{News personalized recommenda- tion operation strategy optimization path}

Any link in the process of personalized news recommendation is very important, which directly affects the user experience. All kinds of problems in personalized news recommendation can only be solved fundamentally from the source. Personalized recommendation has unlimited precision in algorithm recommendation technology, but for the special industry of journalism, the class nature of journalism fundamentally determines the necessity of content control. "Machine replaces labor" not only improves the accuracy of news information dissemination, but also exposes its shortcomings with the fact test. Big data analysis can make up for the shortcomings of manual editing to a certain extent for user behavior analysis, but it can never replace the gatekeeper function of manual editing. As a function of providing information services to users in the development of news information products, personalized news recommendation is a bridge of active communication between the development of news information products and users, so how to build this bridge firmly is a problem that all news information product operators and every news industry practitioner must consider. Therefore, it is a necessary task for the industry to establish a systematic news personalized recommendation operation strategy.

\subsection{Pay attention to content quality control}

It is an unchangeable fact in the content industry that the content is Wang Yongyuan. With the rapid development of Today's Headline, there must be content protection. Technology is armor for content, while content is flesh and blood for journalism. How to attach importance to the quality of news content in the process of news production and dissemination? Users receive personalized recommendation news, and the quality of content directly affects the users' quick positioning and trust in media.

First, it is important to put an end to the low-quality content, and keep strict control by editors.

First of all, the information that users come into contact with in personalized news recommendation includes four parts: source, title, abstract and title. The more authoritative the source, the easier it is for the audience to accept and trust its content. In personalized news recommendation, the proportion of self-media content should be appropriately reduced, and authoritative media news reports should be taken as the main source to fundamentally ensure the authenticity and professionalism of news information. Secondly, in the secondary dissemination, the title should conform to the objective facts, not just to attract users' attention. The perfect title can not only ensure the objectivity of news information itself, but also enhance users' desire for its secondary dissemination.

In addition, news content should be diversified. Personalized news recommendation is not only a single recommendation for information, but also a hot recommendation for the pages of news information products. Diversified and rich page recommendation and single recommendation can attract more users to use their products. The quality of news content is a key issue that must be faced and paid attention to in every era, especially with the blessing of modern technology. The defects of technology need to be perfected by content, and with the improvement of users' own quality, the requirements of content dissemination are also increasing day by day, so it is even more necessary for journalists to improve themselves and provide better news reports, instead of just considering traffic indicators. Content quality has been improved, and traffic can be guaranteed.

Second, it is important to combine localized new with scene recommendation.

Localization of regional news should also be the 
key point of its breakthrough, and news localization mainly refers to recommending social news and peoples' livelihood news in relevant regions according to the location standard of the users' region. The biggest advantage of localized content recommendation is that it narrows the distance between users and can provide users with accurate life news information and big and small things around users. For localized news content recommendation, the most important thing is to accurately locate the user's location information. It is based on LBS technology, which obtains the user's geographic location information through operators and quickly matches the news content for recommendation. For localized news recommendation, it is the best effect to achieve the perfect scene matching with the environment and information needed by users, which will greatly enhance the position of news information products in users' minds. That is to say, the information dissemination of mobile media must match the mobile space, and the user's scene movement in the space also adds a lot of difficulties to news recommendation. Therefore, it is particularly important to combine the location information discrimination technology with the manual recommendation time judgment standard for the localized dissemination of scene-based news.

\subsection{Improve the complex method recom- mendation technology}

Algorithm recommendation saves the audience from the information ocean, and gradually falls into the information cocoon room, which has become the biggest problem faced by recommendation algorithm. Heterogeneity of media production mode. To avoid extreme transformation requires not only strict editing control, but also the operation strategy of the algorithm is one of the important paths. From the update of user information database and the advantages of different algorithms, it is summarized from two aspects:

The first is constantly update the database of user portraits.

Personalized news recommendation often focuses on algorithm recommendation in content selection, so for users, their hobbies and interests are changeable. Therefore, in the technology-based recommendation mode, the analysis data of user behavior should be a variable, rather than remain unchanged after the user portrait is created.
All kinds of client products downloaded by users in the Internet ocean are data that should be paid attention to by algorithm recommendation technology. The prediction of users' intention involves all data information in social networking, lies in the comprehensive analysis of users' social behavior, and is related to the establishment of all subsequent personalized news recommendation strategies. Therefore, it is a huge data analysis process to analyze the user's browsing time, frequency, time and content, and it is also a continuous improvement process, that is, the user's portrait is constantly updated.

Secondly, it is important to combines a variety of algorithms to make up for the shortcomings.

There are many types of algorithm recommendation technology, and a single algorithm recommendation can not fully meet the changing needs of users. All news and information products should integrate the advantages of various algorithm recommendations and adopt a comprehensive method of various algorithm technology modes to learn from each other's strengths, so as to cope with various technical recommendation problems and meet the information needs of more users. At the same time, for the technical development practitioners, it is also very important to study the technical mode that is more suitable for news information recommendation. The details and directions should be considered, such as the research on label refinement or mode diversification, and the establishment of a more comprehensive portrait database for user information. In the news and information product market, there are differences in algorithm technology among different products. To sum up, technology is not static, and it is not omnipotent. Any technical algorithm has drawbacks. Only by developing and constantly improving the algorithm technology suitable for products can the technical advantages be brought into full play.

\subsection{Timely upgrading of products}

The foundation of the existence of any Internet product lies in users, whose consumption, reading, purchasing and other behaviors are the foundation of its existence. In the process of product operation, users have an attitude of expectation and trust from the beginning of downloading, and any details of news recommendation are extremely important, no matter from the appearance or content composition of the product itself. Considering 
problems from the user's point of view can enhance the two-way communication between products and users to the greatest extent. Because as an aggregate, the audience are rich and diverse individuals, the user research of product operators should not be limited to the user research between products, but should also be based on the dynamic trend of users in the whole related Internet content industry, and conduct regular user analysis to formulate the most reasonable user operation mode.

The first is page interaction design optimization.

For personalized news recommendation, while enhancing the communication effect, there is a great communication risk. Whether it is news headlines, news content or news recommendation, all kinds of elements determine the user's reading experience, and users may uninstall because of small error details. Therefore, in the process of actively "selling” news information products, we should pay attention to the user experience, and on this basis, develop products, improve products, optimize products, and provide users with better services. Different products have different interactive design styles, and the optimization process is also a dynamic process of continuous improvement. Therefore, it can be often seen that the new version of the product releases reminders, which are proof of continuous optimization of functions.

The second is the analysis of competing products is normalized.

It is important to keep abreast of competitors' products and market trends. If the data mining channels are reliable and stable, the strategic intention and the latest adjustment direction of the other party can be judged according to relevant data information, and the analysis of competing products can be normalized by comparing its own shortcomings in the operation process. Its purpose is not to study the advantages and disadvantages of competing products, nor how products compete with competing products, nor to prove that the operation scheme is correct or wrong, but to analyze competing products is to predict, observe the past and predict the possible future path of competing products, face up to the present situation of competing products and plan the most reasonable development path for them. For news and information products, the performance of information service is strict, and the competition of news and information products in the market is fierce. Whoever can provide personalized news for users and give the best experience will be the winner.

\subsection{Industry resource advantage sharing}

As far as aggregate news and information products and commercial website clients are concerned, news reports are not their advantages, while as far as traditional media are concerned, communication channels and recommendation technologies are at a disadvantage. Therefore, the sharing of resources in the industry is particularly important. The news and information products under the traditional media need to be spread in a wider range by means of channels, and the aggregated news media needs the supply of high-quality content from the traditional media. Therefore, the two complement each other and need more cooperation while competing.

In addition, intra-industry resource sharing also includes sharing resources of all social products related to users' interests in the Internet industry. The emergence of social products greatly shows the freedom of users on the network platform. Nowadays, social products are not only the information source of news reports, but also can support the whole news reports. Today, when everyone has a microphone, more and more people make comments and opinions on the network platform, then any browsing information and attention information of netizens on the social platform and the stay time of certain content can be used as the data standard for personalized recommendation of news and information products. Therefore, proper sharing of data resources in the Internet industry and sharing in accordance with legal standards can be allowed, which is an important way to help personalized news push more accurately.

It is undeniable that the development of technology has contributed to the news industry, and from a certain point of view, it has revolutionized the news industry. Advantages outweigh disadvantages, but disadvantages cannot be ignored. The integration of technology and news is a booster for news industry, but for journalists, professional requirements seem to be decreasing, which also brings a sense of crisis to traditional news media workers. This triggered the loss of a large number of professionals in the current news industry. Some people started self-media and others turned to commercial media. To a certain extent, this change is not the sharing of talents, but reflects people's neglect of content control from the side. In the era of big data and technology, content is 
equally important. Checking the content of news communication is the rule that the news industry needs to establish, and it is also the order that our whole society needs to establish. For aggregate news and information products, it is necessary to adhere to the operation mode of "algorithm recommendation + user autonomy + editing control" in order to recommend news with the most accurate and good content quality. Under such a recommendation principle, the news can be truly recommended in a personalized way, and the narrow view of developing the news industry only by technology can be avoided. No matter how the times develop and artificial intelligence is outstanding, manual editing is always indispensable in the content era, and the control of content cannot be lost, which is determined by the authenticity of the news industry and required by the values in the development of the times.

\subsection{Learn from foreign advanced experience}

In China, news and information products mainly based on algorithm recommendation have developed rapidly in the past two years and gained market share. Similarly, in the United States, the trend of personalized push is also popular. In this matter, the global news industry seems to be facing the same problems and puzzles. How to make personalized recommendation the best? How to make users accept personalized recommendation better?

Both News Republic and Today's Headlines are also faced with the contradiction between technology and content. From the aspect of content output, neither of them has the right to edit and edit, so they are faced with the same problems in the process of developing self-media and content handling. Although the protection of property rights in China has made initial progress in the past two years, it is still not as important as that in western countries.

Apart from copyright issues, there are lot to learn from advanced experience in the expression of content recommendation. For example, all kinds of news and information products in the United States combine their audience positioning and brand characteristics to innovate the recommendation form. That is to say, as a news recommendation, which seems to only shoulder the function of news event reporting and dissemination, it has strong autonomy to a certain extent. In a great sense, it is an active display of media style positioning and a further innovation of brand content. When the user receives the general text recommendation, even if the text content is interesting and interesting, only the text will lose more than half of the reading interest. Then, combined with the domestic news information product market, not only the active recommendation content style can be learned, but also various recommendation attempts can be launched, such as branded HS news reports, short video news, comic news, etc., which are not limited to the simple reporting of news events. Although personalized recommendation pays attention to the immediacy of news, it does not mean that every piece of news should be expressed in such a form. Various forms of expression do lead to information lag in speed, and innovative content can be personalized push according to the actual operation situation.

\subsection{Improve the media literacy of users}

Users' needs are ever-changing, their quality varies, and their media literacy is also different. In today's era, daily news information is mixed, true and false. For users themselves, it is extremely important to strengthen their ability of content discrimination and media literacy. Due to the influence of various factors, the channels for users to improve their media literacy should be strengthened from the perspectives of individuals and society.

\section{Conclusion}

In a word, in the era of mobile Internet, news information products, as the content production platform and channel communication platform of news industry, play a vital role. From the perspective of communication, news and information products play the role of commander-in-chief. What kind of content, when, in what way and to which users will directly affect the communication effect. So how to give full play to the advantages of such communication effect, pay attention to the quality of communication content, and at the same time, and how to spread it to users more accurately become important problems urge to be solved. In today's era of information explosion, they are particularly important. Personalized recommendation of news under the recommendation of algorithm has become the operation mode with the lowest cost and the most direct communication effect in the operation process of news infor- 
mation products. Customer first has become the foundation of the development of news media industry and even the whole Internet industry. However, blindly taking this idea as the only standard of news media content dissemination and excessively relying on personalized recommendation technology will inevitably fall into technical quagmire and provide a living soil for low-quality content.

\section{References}

1. Tan B. International Information (in Chinese). Ar- chives Management 2020; (5): 1.

2. Yang L. Application, influence and reflection of personalized recommendation in mobile news communication (in Chinese). News and Communication Review 2020; 73(2): 47-58.

3. Ma J. Research on user experience of news and information app (in Chinese). Changchun University of Technology; 2019.

4. Hu W. The ethical dilemma of news algorithm push and its countermeasures (in Chinese). Changchun University of Technology; 2019.

5. Zhang X, Li J. Predicament, outlet and prospect of news algorithm push (in Chinese). Editorial Friend 2019; (4): 86-90. 